\title{
Linx
}

Revue des linguistes de l'université Paris X Nanterre

$77 \mid 2018$

Regards croisés sur le futur en français et dans différentes langues romanes

\section{Premiers pas dans le futur. Hypothèses dialogiques}

\section{Anne Salazar Orvig}

\section{(2) OpenEdition}

\section{Journals}

Édition électronique

URL : http://journals.openedition.org/linx/2722

DOI : $10.4000 / \operatorname{linx} .2722$

ISSN : 2118-9692

Éditeur

Presses universitaires de Paris Nanterre

\section{Édition imprimée}

Date de publication : 30 octobre 2018

Pagination : 65-92

ISBN : 978-2-84016-329-9

ISSN : 0246-8743

Référence électronique

Anne Salazar Orvig, "Premiers pas dans le futur. Hypothèses dialogiques », Linx [En ligne], 77 | 2018, mis en ligne le 30 avril 2019, consulté le 03 mai 2019. URL : http://journals.openedition.org/linx/2722 DOI : 10.4000/linx.2722

Ce document a été généré automatiquement le 3 mai 2019.

Département de Sciences du langage, Université Paris Ouest 


\title{
Premiers pas dans le futur. Hypothèses dialogiques
}

\author{
Anne Salazar Orvig
}

\section{Introduction}

1 Cet article s'intéresse aux usages du futur chez les adultes et aux conditions dialogiques qui pourraient accompagner son acquisition par les jeunes enfants. Dans le processus d'appropriation de sa langue maternelle, le jeune enfant doit à la fois acquérir des formes, les valeurs ou significations de ces formes et leurs usages. Si les approches de type innéiste/générativiste (cf. Jakubowicz, 1995 ; Lust, 2006) postulent une continuité entre langue adulte et langue de l'enfant, des mécanismes prédéfinis qui expliquent le développement des productions enfantines et une extériorité des usages par rapport au système linguistique, les approches fonctionnalistes (MacWhinney, 2004), interactionnistes ou dialogiques (Bruner, 1983 ; François et al., 1984 ; Salazar Orvig, 2017) et/ou basées sur l'usage (Tomasello, 2003; Tomasello, 2004 ; Lieven et Tomasello, 2008) ont en commun de postuler le fondement communicationnel du langage, le rôle actif de l'enfant dans la construction/élaboration de sa langue et, de façons diverses, l'importance de l'expérience communicative de l'enfant. Cette dernière peut être conçue comme l'exposition à des usages linguistiques, les formes linguistiques adressées à l'enfant, les patterns ou constructions les plus fréquents ou comme l'inscription dialogique de l'adulte et de l'enfant dans des situations communicatives. Dès lors le rapport entre usage et système linguistique se trouve inversé, l'usage n'étant pas uniquement la mise en fonctionnement de la langue mais bien le matériau à partir duquel l'enfant s'approprie la langue, construit un système de formes et de règles, et les significations des unités linguistiques et des structures. 


\subsection{Quelques repères sur l'acquisition du futur}

2 En ce qui concerne les temps verbaux, les diverses études se situant dans des approches fonctionnalistes et/ou basées sur l'usage, montrent que les enfants construisent les paradigmes morphologiques de façon progressive, à partir d'occurrences isolées de lexèmes référant à des processus (proto-verbes) ${ }^{1}$ passant de l'usage d'une seule forme par verbe (Pizzuto et Caselli, 1994 ; Dressler et al., 2003) à une relative productivité. La forte association entre lexèmes verbaux et flexions ne correspond d'ailleurs pas uniquement au processus de mise en place d'un paradigme morphologique. Elle reflète plutôt les configurations des usages adultes desquels les enfants tendent à se rapprocher (Wilson, 2003; Pine et al., 2008; pour les usages du futur et du conditionnel en français Morgenstern et al., 2010 ; Morgenstern et Parisse, 2012).

3 Sur le plan sémantique, la construction des valeurs temporelles est également un processus long, qui dépasse largement la petite enfance (Bronckart et Sinclair, 1973). Les différents travaux sur l'acquisition des temps insistent sur le fait que les premières formes des enfants restent ancrées dans le hic et nunc de l'énonciation (ce qui est aussi une propriété des dialogues auxquels ils participent, cf. Ninio et Snow, 1996 ; Veneziano, 2001) et que leurs valeurs seraient plus aspectuelles que temporelles (Antinucci et Miller, 1976 ; Sabeau-Jouannet, 1977 ; Bronckart et al., 1983).

4 Les auteurs s'accordent pour constater l'antériorité des formes du futur périphrastique sur les formes du futur simple. En français, les premières occurrences du futur périphrastiques sont constatées autour de 2 ans (Sabeau-Jouannet, 1977; Bassano et al., 2004 ; Morgenstern et al., 2010). Par ailleurs, Bassano et al. (2004) relèvent que la forme aller+infinitif apparaît après la forme modal+infinitif, légèrement plus précoce. Ces deux structures sont elles-mêmes précédées par des précurseurs, l'infinitif seul («mettre là » pour « (je veux/vais mettre là ») ou filler+infinitif « eum /pas percer » pour «je veux pas percer »), interprétable en contexte avec des valeurs similaires.

5 Sur le plan sémantique, les travaux présentent moins de convergence. Pour SabeauJouannet, les premiers usages du futur périphrastique seraient de type aspectuel ou modal et «aucune forme du futur périphrastique n'est attestée sans le début de la réalisation matérielle dans le comportement de l'enfant lorsque celui-ci est agent » (1977 ; 194). Morgenstern et al. (2010) notent cependant des usages en discontinuité avec le moment d'énonciation, tout en constatant que les usages en continuité sont plus nombreux et plus précoces.

6 Le futur simple est plus tardif. Sabeau-Jouannet ne le relève pas avant 2;9 et dans le corpus de Morgenstern et al., les premières occurrences peuvent se situer au plus tôt à $2 ; 4$, pour l'enfant la plus précoce et 2;11 pour le plus tardif. Alors que Sabeau-Jouannet insiste sur la coexistence des marqueurs adverbiaux, qui conféreraient aux premiers usages du futur simple sa valeur temporelle, Morgenstern et al. soulignent la prééminence des valeurs de discontinuité temporelle par rapport au moment de l'énonciation. Cette discontinuité peut se combiner avec des modalités, qui sont souvent porteuses de la flexion verbale, comme dans l'exemple suivant : 
(1) Exemple de Madeleine 2;7

${ }^{*} \mathrm{CHI}$; il a de grand[/] grand [/] grand du coup faut [///] faudra lui acheter du [//]

des lunettes.

(Morgenstern et al., $2010: 11$ )

7 Morgenstern et al. (2010) ont également mis en évidence que les enfants présentent des profils similaires à ceux de leurs interlocuteurs adultes aussi bien dans la proportion d'occurrences des deux formes de futur que dans celle des valeurs de continuité et de discontinuité. Ils signalent que chez les adultes en dialogue avec les enfants, les usages du futur simple sont quasi systématiquement associés à des marquages temporels, modaux ou argumentatifs. Il en est de même pour la valeur discontinue du futur périphrastique

\subsection{Quelle approche pour rendre compte de l'acquisition du futur?}

Ainsi, la question se pose non seulement de la mise en place du paradigme des flexions verbales, au sein du processus de construction de la grammaire, mais aussi de comment l'enfant s'approprie les valeurs (temporelles et/ou modales) des tiroirs verbaux, ce "comment» pouvant à son tour être décliné en deux questions complémentaires: « comment » sur le plan de l'évolution des valeurs, et « comment » sur le plan processuel, quels sont les mécanismes en jeu, d'où puise-t-il ces valeurs, comment les intègre-t-il ? Alors que l'acquisition des formes se laisse expliquer par des processus de reprise et d'analyse distributionnelle fonctionnelle (Tomasello, 2003), l'appréhension du sens par l'enfant est l'objet de bien plus de débats. Les approches socio-pragmatiques et interactionnistes ont montré que les enfants intègrent des formes et leur donnent sens à travers leur compréhension des dialogues dans lesquels ils sont impliqués. Les travaux de Tomasello et son équipe (cf. Tomasello, 2004) ont montré comment de très jeunes enfants saisissent le sens de nouveaux noms ou verbes à partir de leur compréhension de l'intention de l'adulte dans une séquence interactive donnée. Cependant, comme le signale Nelson (2007), la tâche n'est pas du même ordre dès lors qu'il s'agit pour l'enfant de saisir des concepts abstraits non saillants dans l'interaction. Dans une étude sur l'acquisition de termes temporels («before», " after») et causaux (" 'cause ») chez une petite fille, Levy et Nelson (1994) ont montré que l'acquisition du sens de ces mots est précédée par l'appréhension par l'enfant de leur usage en discours, usage qu'elle reproduit dans des formats syntaxiques contraints et en lien avec des thématiques privilégiées. Ces premières reprises sont adéquates du point de vue contextuel mais non du point de vue sémantique. Confronté à son propre usage et aux réactions des adultes, l'enfant va, peu à peu, élaborer un sens de plus en plus abstrait et conforme au sens conventionnel. Ainsi, l'enfant saisit d'abord un « quand utiliser la forme » avant de saisir la signification elle-même. Ou pour reprendre la formulation des auteures, elle en saisit la dimension pragmatique avant la dimension sémantique.

9 D'une façon générale, les approches interactionnistes et dialogiques considèrent que les acquisitions linguistiques et, $a$ fortiori, celles relevant du sens et de la référence se font dans le cadre des jeux de langage (Wittgenstein, 1961) dans lesquels l'enfant les rencontre. C'est dans cet esprit que la présente étude sur l'acquisition du futur a été envisagée. Il ne s'agit donc pas de rendre compte des premières productions enfantines $\mathrm{du}$ futur mais d'explorer les formes, valeurs et usages du futur auxquels les enfants sont confrontés dans les échanges dialogiques auxquels ils participent, et de tenter de 
comprendre ce qui dans cette expérience pourrait leur permettre d'en appréhender et (re)construire progressivement les valeurs.

10 Afin d'identifier les contextes dans lesquels les jeunes enfants rencontrent les usages des deux formes du futur, j'ai travaillé sur un corpus ${ }^{2}$ transversal de dialogues adulte-enfant. Il s'agit des dialogues de 24 enfants, âgés entre $1 ; 10^{3}$ et 2;6 ans, enregistrés dans un contexte familial, le plus souvent avec leur mère, mais aussi avec leur père, des frères et sœurs ou l'observatrice ${ }^{4}$. Ces échanges se déroulaient lors d'activités familières telles que des goûters, des lectures ou de jeux (puzzle, loto, maisons de poupées, constructions). Dans la mesure où certains enfants ont été enregistrés plusieurs fois (de 2 à 5 séances), le corpus comporte 42 séances. Cette première étude a été complétée par une exploration qualitative de six séances tirées du corpus longitudinal d'Anaé entre 2;2 et 3;5 (Morgenstern et Parisse, 2012).

11 L'analyse du corpus transversal a été conduite en trois temps : a) repérage des références au FUTUR ${ }^{5}$ et identification des formes $\left.(\$ 2) ; b\right)$ codage des valeurs illocutoires des énoncés dans lesquels ces formes sont utilisées ( $(3)$; c) étude des contextes dialogiques d'usage du futur simple (\$4).

\section{L'expression du FUTUR dans des dialogues adulte- enfant}

Dès lors qu'on ne postule pas une grammaire innée ni de continuité entre le sémantisme chez l'enfant et chez l'adulte, on ne peut présupposer de relation biunivoque entre forme et sens ; tout d'abord parce que, dans le processus d'acquisition de leur langue, les enfants tendent à exprimer des nouvelles significations avec les moyens linguistiques qu'ils maîtrisent (Slobin, 1973) ; surtout parce que si les formes du futur périphrastique et du futur simple présentent chez l'adulte des gammes de valeurs en intersection partielle (Branca-Rosoff, 2009; Azzopardi, 2011; Abouda et Skrovec, 2017, entre autres), les adultes expriment également le temps FUTUR avec d'autres tiroirs verbaux (Gosselin, 1996). La première analyse a donc consisté à repérer sur la base d'un critère référentiel toutes les formes verbales, à l'exception des impératifs, référant à un état, un événement ou un 
processus ultérieur au moment de l'énonciation, quelle que soit la distance temporelle par rapport à ce moment de l'énonciation.

\begin{tabular}{|c|c|c|c|c|c|c|c|c|}
\hline & \multicolumn{4}{|c|}{ EXPRESSION DU FUTUR } & \multirow[t]{2}{*}{$\begin{array}{l}\text { AUTRES } \\
\text { TEMPORELLES }\end{array}$} & \multirow[t]{2}{*}{ INDÉC } & \multirow[t]{2}{*}{$\begin{array}{l}\text { VERBES NOMBRE } \\
\text { TOTAL }\end{array}$} & \multirow{2}{*}{$\begin{array}{l}\text { ENONCES } \\
\text { NOMBRE } \\
\text { TOTAL }\end{array}$} \\
\hline & $\begin{array}{l}\% \\
\text { total }\end{array}$ & médiane & $\min$ & $\max$ & & & & \\
\hline Enfants & 14,5 & 10,9 & 0 & 37,7 & 84,9 & 0,6 & 1604 & 4843 \\
\hline Parents & 22,1 & 20,0 & 4,7 & 44,0 & 77,9 & 0,0 & 5687 & 7796 \\
\hline Total & 20,5 & & & & 79,4 & 0,1 & 7291 & 12639 \\
\hline
\end{tabular}

Tableau 1 Pourcentage de références au FUTUR dans les dialogues adulte-enfant.

Le tableau 1 montre que la référence au FUTUR occupe globalement $20 \%$ des verbes, les enfants en produisant par ailleurs notablement moins que les adultes. Les valeurs médianes, minimales et maximales dans le tableau 1 montrent par ailleurs la très grande variabilité dans nos données. De fait, deux enfants ne produisent aucune référence au futur et sept d'entre eux ne le font que dans une de leurs séances. De même, on constate une importante variabilité parmi les adultes: dans 17 séances (impliquant 14 adultes différents) la mention du FUTUR n'atteint pas $15 \%$ des verbes alors que dans d'autres elle peut s'élever à plus de $30 \%$ des verbes. Même si l'on constate dans les séances des enfants les plus jeunes de faibles pourcentages d'expression du FUTUR, la part globale de cette référence dans le dialogue n'est pas corrélée ${ }^{6}$ avec le développement linguistique de l'enfant mesuré par la longueur moyenne des énoncés (LME).

14 Les expressions ont été ensuite étiquetées en fonction du tiroir verbal utilisé. Cinq catégories de formes ont été relevées. En premier lieu on constate des usages fréquents du présent, comme on peut le voir dans les énoncés d'Ilona (2) ou dans celui de la mère de Julien (3) :

(2) Ilona $2 ; 3^{7}$

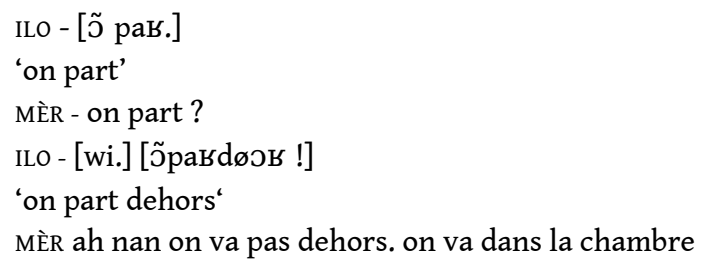

(3) Julien 2;3

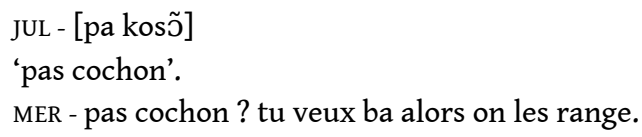

Comme ces exemples le montrent, le présent apparait dans des énoncés de régulation de l'activité et souvent avec une valeur injonctive (voir \$3). Si la plupart des occurrences 
renvoient à des actions devant se réaliser dans un futur immédiat, on peut également constater le recours au présent pour référer à des événements en discontinuité temporelle avec le moment de l'énonciation (même s'ils sont déictiquement déterminés) :

(4) Maxime, 2;3

MER - tu sais où on va ce soir?

16 La deuxième catégorie correspond aux constructions comportant un modal (vouloir, pouvoir, devoir, falloir ...) au présent associé à un infinitif (5) ou, chez les adultes, à une subordonnée (6) :

(5) Julien 2;3

MÈR - on range dans la boite. d'accord

JUL - [ว̃pø lьãze]

'on peut $1(\mathrm{e})$ ranger'

\section{(6) Mathilde 2;4}

MÈR - tu veux qu'ils montent dans le train?

17 Une troisième catégorie correspond aux cas où un infinitif est produit sans auxiliaire modal (7) ou dans une proposition infinitivale. Chez les enfants cela peut correspondre en outre à une difficulté à construire un prédicat complexe (8) :

(7) Olga 2;3

Olga et sa mère jouent au loto.

MER - qu'est ce que c'est ça?

OLG - [lə metmetu ?]

'le mettre où ?'

MER - ben tu le mets bah regarde où il est le parapluie.+ sur quelle image il est ?

(8) Elodie 2;3

MÈr - tu vas enlever la voiture ? pourquoi ? regarde+elle avance toute seule

ELo - [nõ mwa ãve]

'non moi en(le)ver'

MÈr - non tu vas enlever $\{\mathrm{xxxx}\}$

La quatrième catégorie correspond aux occurrences du futur périphrastique. Cette forme recouvre des valeurs diverses, depuis la référence à une action ou à un processus imminent (9) jusqu'à des événements distants du moment de l'énonciation (10) :

\section{(9) Arnaud, 2 ;3}

Arnaud joue avec des prises ; sa mère le guide dans ses branchements MÈR - tu vas la brancher là-bas

ARN - <soupire>

MÈR - dans la lampe ? tu la mets dans la lampe?

(10) Théo - 2;3

MÈR - tiens regarde c'est là qu'on va aller. <la mère montre un dépliant à Théo>

THE - $\{\mathrm{Xxx}\}$

MÈR -MER - on va aller à Eurodisney. et qu'est- ce qu'on va rencontrer là-bas ? 
19
12), ces formes ne renvoient pas nécessairement à une ultériorité dissociée du moment de l'énonciation :

\section{(11) Arnaud 2;3}

MÈR - attends, je te donne un petit morceau $\mathrm{p}(\mathrm{u})$ is t'en reprendras après

(12) Pauline 2;3

MÈR - regarde il en reste un p(e)tit peu. <mère racle l'intérieur du pot avec la cuillère>

PAU - [е арь

'et après y aura d'autres'

\begin{tabular}{|l|l|l|l|l|l|l|l|}
\hline & Présent & Infinitif & Modal +V & Futur P. & Futur S. & Autres $^{8}$ & Nombre Total \\
\hline Enfants & 30,0 & 20,6 & 27,5 & 21,5 & 0,4 & 0 & 233 \\
\hline Adultes & 35,7 & 1,0 & 28,3 & 31,3 & 3,6 & 0,2 & 1258 \\
\hline Total général & 34,8 & 4,0 & 28,2 & 28,8 & 3,1 & 0,1 & 1491 \\
\hline
\end{tabular}

Tableau 2 Distribution (en \%) des tiroirs verbaux dans les cas de référence au FUTUR.

21 Le tableau 2 présente la distribution des différentes formes pour les enfants et les adultes. Un premier constat s'impose. Chez les adultes, près de deux tiers de références au FUTUR sont portées par des tiroirs verbaux autres que les deux futurs. Cette dominance atteint $78 \%$ chez les enfants. En outre, on constate une relative similitude dans le recours au présent et aux constructions modales chez les enfants et chez les adultes. Cependant, comme l'a souligné Bassano (2004), chez les enfants la proportion relativement importante de formes de l'infinitif correspond probablement à des précurseurs de la construction modale ou du futur périphrastique (8).

La grande variabilité des dialogues se confirme sur ce plan. En effet la part des deux futurs peut aller de 0 à $67 \%$ des références au FUTUR. Quand une seule des deux formes est utilisée c'est celle du futur périphrastique (39 séances). En revanche, seulement 15 adultes (dans 21 dialogues sur 42) et un seul enfant (12) $)^{9}$ présentent des occurrences du futur simple. Cette variabilité ne semble pas pouvoir s'expliquer par une adaptation des adultes au développement des enfants. Si la part d'usage des tiroirs verbaux du futur chez les enfants est corrélée à celle des adultes ( $\mathrm{r}: 0.42, \mathrm{p}=0.017)$, elle ne l'est ni à l'âge ni au développement linguistique. D'ailleurs, les 21 dialogues présentant des futurs simples n'impliquent pas nécessairement les enfants les plus âgés.

Une part de ces différences dépend, probablement, de l'effet de styles individuels des parents et/ou des enfants. C'est une question à laquelle on ne peut pas répondre ici car les données par individu sont insuffisantes. En revanche, une autre part de ces différences pourrait être expliquée par l'effet du type d'interaction et des genres dans lesquels parents et enfants sont impliqués (Fleury et Branca-Rosoff, 2010). Ce à quoi s'ajoute, nécessairement, la dynamique des dialogues. Dans ce qui suit j'explore les effets des interactions à travers deux pistes complémentaires, la dimension illocutoire et les séquences dialogiques ${ }^{10}$. 


\section{L'expression du futur est-elle associée à des valeurs illocutoires préférentielles?}

Alors que la question de la tension entre valeurs temporelles et valeurs modales (déontique, épistémique, volitive...) parcourt la littérature sur le futur (Abouda et Skrovec, 2015; Alvarez Castro, 2010; Azzopardi, 2011; Branca-Rosoff, 2009), peu de travaux se sont intéressés à ses affinités avec la valeur illocutoire des énoncés. Or, les valeurs ou usages modaux du futur sont à leur tour fortement associés (comme constitutifs ou comme effets) à la dimension illocutoire des énoncés : par exemple, Searle (1972) considère comme constitutive des actes directifs (ordre, demande, prière) et des actes promissifs (promesses, menaces, engagement) la condition de contenu propositionnel selon laquelle l'énoncé prédique une action future du destinataire, pour les directifs, ou du locuteur, pour les promissifs. De même, l'expression par le locuteur de son souhait de voir une action réalisée (condition de sincérité) ou l'expression de la possibilité ou la capacité du destinataire à réaliser une action (condition préparatoire) constituent des indices forts de l'existence d'une requête indirecte (Searle, 1979).

Dans le cadre d'une étude sur le développement de la compréhension des promesses chez le jeune enfant, Bernicot et Laval (2004) se sont interrogées sur les indices linguistiques permettant au jeune enfant de comprendre un énoncé de forme déclarative comme une promesse. Leur recherche a montré que les enfants évoluent d'une interprétation fondée sur des facteurs contextuels à une interprétation utilisant des indices linguistiques. Ainsi les enfants plus jeunes (trois ans) identifient les promesses en se basant sur le contexte et en particulier en reconnaissant la condition préparatoire sur la base de situations prototypiques. Si les enfants de six ans identifient le futur périphrastique comme un marqueur de promesse, leurs réponses sont également fortement facilitées par le contexte. Seuls les enfants de neuf ans semblent s'être autonomisés des informations contextuelles et se basent sur les marques linguistiques (futur périphrastique et futur simple) pour reconnaître la valeur illocutoire de l'énoncé. Cette étude met ainsi en évidence la façon dont pour les jeunes enfants le marquage linguistique est ancré dans des contextes prototypiques.

La question s'imposait donc d'évaluer l'existence ou non d'affinités fortes dans le discours parental (et des enfants) entre les formes d'expression du FUTUR et des valeurs illocutoires spécifiques: par exemple, le présent avec valeur de FUTUR est-il utilisé de façon préférentielle pour les ordres ou les requêtes, et le futur périphrastique pour les promesses? Cette analyse a été conduite à partir d'une taxinomie des actes de langage adaptée ${ }^{11}$ de celle de Searle (1979). Les catégories suivantes ont été identifiées :

PROMISSIFS : J'ai considéré comme promissifs (dans leurs deux polarités de promesse et menace) des actes engageant le locuteur vis-à-vis du destinataire (qui souhaite ou craint l'action à venir).

(13) Mathilde, 2;4

MAT - [abobo lapat]

'a bobo la \{patte/table\}'

MER - qui a bobo?

PER - la table

MAT - [akכь] <elle donne un rouleau de scotch à son père> 
'encore'

PER - ah oui mais la table elle n'a pas bobo Mathilde. ça c'est pour coller des choses.

on n'en a pas besoin. ça c'est pour coller des choses

MAT - $[\tilde{\varepsilon}]$

'hein'

PER - oh la la c'est cassé ! bon. papa il va le réparer.

\section{(14) Clément 2;3}

PER - tu manges ton gâteau. si tu le manges pas je vais le manger celui-ci s'en porte garant.

(15) Séréna 2;3

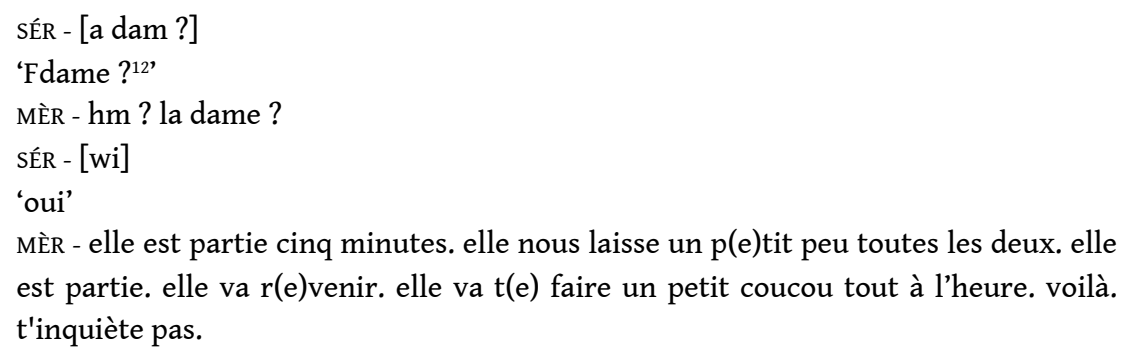

La catégorie des directifs de Searle s'est avérée trop vaste et hétérogène quand on traite de dialogues (Kerbrat-Orecchioni, 2001) et plus particulièrement de dialogues familiaux. Trois catégories distinctes ont été ici considérées :

DEMANDES : Il s'agit de la catégorie la plus prototypique des directifs searliens. On y inclut les requêtes, les ordres, les prières, les instructions, les interdictions et les demandes de permission. Dans ces différents cas, le locuteur demande à son interlocuteur d'accomplir ( 16) ou au contraire de ne pas accomplir (17) une action, voire de lui permettre d'accomplir une action (18). Cette catégorie regroupe les requêtes directes et les requêtes indirectes.

(16) Iris $1 ; 11$

Iris et son père jouent avec un Monsieur Patate, bonhomme à construire PER - $\mathrm{tu}^{13}$ lui remets la bouche et tout ça

(17) Julien 2;3

Julien se rapproche de la caméra, posée sur un trépied

JUL - [se a madam ]

'c'est à madame'

MÈr - c'est à la dame oui. donc on on ne touche pas à la caméra.

(18) Ilona 2;3

MÈr - tu veux d'la brioche?

MÈr - allez vas-vas t'asseoir pour la manger. vas t'asseoir toute seule! maman elle

peut goûter?

ILO - [nã !]

'non'

PROPOSITIONS : cette catégorie, certes hétérogène, regroupe des énoncés se situant à michemin entre les requêtes et les promissifs. Les propositions (19) sont produites souvent 
sous forme interrogative et sollicitent l'interlocuteur sur ses désirs et souhaits sans instaurer pour autant une obligation de faire. Par ailleurs, l'état psychologique de l'allocutaire n'est pas nécessairement connu. En revanche, comme pour les promissifs, la proposition peut également impliquer une projection du locuteur dans la réalisation de l'action:

\section{(19) Alice1 1;11}

Alice et sa mère jouent à la poupée

MÈr - tu as vu quand elle est couchée elle ferme les yeux. elle est contente

ALI $\{X\}$

MÈr - ça y est elle a bien mangé. tu veux qu'on l'assoit pour la peigner ?

QUESTIONS: les questions se distinguent des autres directifs par le fait que le comportement sollicité est une réponse verbale (Kerbrat-Orecchioni, 2001). Que les questions soient de nature informationnelle ou méta-énonciative, tous les types (questions ouvertes, catégorielles, ou fermées, voire les demandes de confirmation) ont été regroupés ici.

(20) Arnaud 2;3

Arnaud joue à brancher des prises. Sa mère le guide.

MÈR - tu vas brancher ça maintenant

ARN - [a brode a pri u lø.lø lø lø]

'F \{branché/er\} Fpri(se) ou le le el le'

MÈR - oui. voilà. très bien. et où est-ce que tu le mets ?

(21) Julien

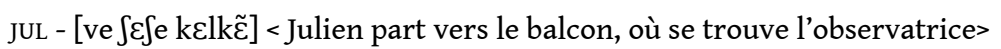

'vais che(r)cher quelqu'un'

MÈr - qu'est-ce (que) tu vas chercher?

ASSERTIFS : On considère comme assertif tout énoncé décrivant un état de choses, à propos duquel s'engage le locuteur. Portant sur le fUtur, qui implique l'ordre du possible, la question de la valeur de vérité est suspendue et le caractère assertif résulte d'un effet pragmatique (Alvarez Castro, 2007). Ainsi, les énoncés classés comme des assertions correspondent à des prédictions, des annonces d'action ou d'activité, ou encore des avertissements. Certains de ces assertifs sont - comme le soulignait Austin (1970) proches des promissifs ; entre l'annonce de ce que l'on compte faire et l'engagement visà-vis de quelqu'un, la limite est ténue ; comme elle l'est entre l'avertissement et la menace. J'ai considéré comme assertifs les énoncés pour lesquels ne se vérifie pas la condition préparatoire du promissif (le récepteur souhaite - ou craint que l'acte futur se réalise). Ainsi, même si les deux énoncés de l'adulte dans les exemples (13) et (22) se ressemblent sur le plan formel, ils ne correspondent pas au même acte illocutoire.

(13) Mathilde, 2;4

(...)

PER - ah oui mais la table elle n'a pas bobo Mathilde. ça c'est pour coller des choses.

on n'en a pas besoin. ça c'est pour coller des choses

MAT - $[\tilde{\varepsilon}]$

'hein'

PER - oh la la c'est cassé ! bon. papa il va le réparer. 
(22) Ilona 2;3

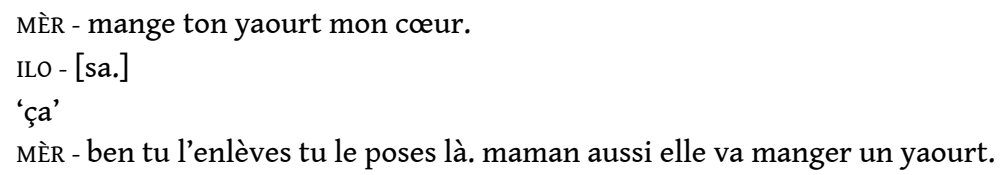

Dans l'exemple (13), l'énoncé du père « bon. papa il va le réparer » a été codé comme une promesse parce que dans les énoncés précédents l'enfant réclame qu'on s'occupe de la table (et de son bobo). Même s'il considère que la table n'a pas bobo, le père s'engage à s'en occuper. L'énoncé de la mère dans (22) a été au contraire considéré comme une assertion parce que celle-ci informe l'enfant de ce qu'elle va faire. Cette action future n'est ni souhaitée ni crainte par l'enfant.

Le tableau 3 présente la distribution des actes de langage pour chaque expression du FUTUR pour les parents et pour les enfants

\begin{tabular}{|c|c|c|c|c|c|c|c|}
\hline & Futur P. & Futur S. & Infinitif & Modal +V & Présent & $\%$ Total & Nbre Total \\
\hline \multicolumn{8}{|l|}{ Parents } \\
\hline Promissifs & $98(25 \%)$ & $28(62 \%)$ & 0 & $2(1 \%)$ & $22(5 \%)$ & 12,0 & 150 \\
\hline Demandes & $46(12 \%)$ & $4(9 \%)$ & $2(17 \%)$ & $113(32 \%)$ & 227 (51\%) & 31,3 & 392 \\
\hline Questions & $56(14 \%)$ & $1(2 \%)$ & $1(8 \%)$ & $86(24 \%)$ & $72(16 \%)$ & 17,2 & 216 \\
\hline Propositions & $28(7,1 \%)$ & $1(2 \%)$ & 0 & $77(22 \%)$ & $74(17 \%)$ & 14,4 & 180 \\
\hline Assertifs & $164(42 \%)$ & $11(24 \%)$ & $9(75 \%)$ & $77(22 \%)$ & $54(12 \%)$ & 25,1 & 315 \\
\hline $100 \%=$ & 392 & 45 & 12 & 355 & 449 & & 1253 \\
\hline \multicolumn{8}{|l|}{ Enfants } \\
\hline Promissifs & 0 & 0 & $2(4 \%)$ & 0 & $1(1 \%)$ & 1,3 & 3 \\
\hline Demandes & $8(16 \%)$ & 0 & $20(42 \%)$ & $41(64 \%)$ & $32(46 \%)$ & 43,3 & 101 \\
\hline Questions & 7 (14\%) & $1(100)$ & $2(4 \%)$ & $1(2 \%)$ & $12(17 \%)$ & 9,9 & 23 \\
\hline Propositions & $1\left(2 \%^{\circ}\right.$ & 0 & 0 & $1(2 \%)$ & $5(7 \%)$ & 3,0 & 7 \\
\hline Assertifs & $34(68 \%)$ & 0 & $24(50 \%)$ & $21(33 \%)$ & $20(29 \%)$ & 42,5 & 99 \\
\hline $100 \%=$ & 50 & 1 & 48 & 64 & 70 & & 233 \\
\hline
\end{tabular}

Tableau 3 Distribution des expressions du FUTUR pour chaque type d'acte de langage, chez les parents et les enfants

Le tableau 3 permet de constater que même si il n'y a pas d'association exclusive entre tiroirs verbaux et actes de langage, chez l'adulte, les formes ne se distribuent pas de façon aléatoire. Le futur périphrastique apparaît surtout dans les assertifs mais il est également proportionnellement plus fréquent dans les promissifs. Corrélativement, promissifs et assertifs sont très souvent associés au futur périphrastique. A l'inverse il est peu utilisé dans les demandes et les propositions. Le futur simple apparait surtout dans les promissifs (même si l'inverse n'est pas vrai, les promissifs ne sont pas exprimés de façon dominante avec le futur simple), puis en moindre mesure dans les assertifs. Les constructions modales, quasi absentes des promissifs, se distribuent de façon presque similaire entre les 
quatre autres types d'actes de langage. Il faut également noter que la part de questions et de propositions parmi les constructions modales est proportionnellement plus importante que leur part dans l'ensemble du corpus, ce qui dénote une association préférentielle. Enfin le présent est préférentiellement associé aux demandes.

Chez les enfants on constate également un usage varié des formes, mais pour des actes de langage moins diversifiés. De façon plus marquée que chez les adultes, les enfants utilisent le futur périphrastique dans des énoncés assertifs. Les demandes dominent pour le présent mais leur proportion n'est pas supérieure à la distribution des demandes dans l'ensemble du corpus. Même si les assertions correspondent à $29 \%$ des présents, cette proportion est bien inférieure à la proportion d'assertifs dans l'ensemble du corpus $(42,5 \%)$. Les constructions modales apparaissent chez les enfants essentiellement dans des demandes. Comme pour le présent, la proportion d'assertions parmi les constructions modales est inférieure à la proportion d'assertions parmi les énoncés des enfants. Les infinitifs se partagent en deux grands groupes, les demandes et les assertions. On peut penser que cela correspond à leur statut de précurseur à la fois du futur périphrastique et des constructions modales.

Ainsi les enfants présentent des similitudes et des différences avec les usages des adultes. D'une façon générale, les enfants mobilisent les mêmes types de formes pour les mêmes fonctions pragmatiques. Les différences pourraient être attribuées non pas au développement linguistique, mais à l'asymétrie dans leurs rôles interactionnels (Ochs et Schieffelin, 1995). En effet, le fait n'est pas que les enfants mobilisent peu le futur pour des promesses ou des propositions, mais plutôt que compte tenu des contextes interactionnels et de leur âge, ils ne se trouvent pas en situation de promettre ou de proposer une activité. De même, le fait que les enfants présentent proportionnellement plus de constructions modales (ou l'infinitif) que le présent pour les demandes peut être expliqué par la position dans le dialogue, qui les amène à être moins injonctifs que les adultes.

39 Ces résultats suggèrent donc que malgré la diversité des usages, les enfants font l'expérience d'associations préférentielles fortes entre certains temps et certains actes de langage. Et que leurs usages, sans en être un calque strict, portent en germe les usages adultes.

\section{L'usage du futur simple en dialogue}

40 Si on peut comprendre comment les enfants saisissent l'usage du futur en association avec des valeurs illocutoires spécifiques, une autre question est celle de savoir comment ils en saisissent, sur le plan sémantique, la dimension temporelle. Pour cela, il est nécessaire d'explorer les contextes où ils l'entendent. Je prendrai, pour cerner cette question, la façon dont se déploie, en dialogue, l'usage du futur simple $e^{14}$. Le futur périphrastique, bien plus fréquent, apparaît déjà dans des contextes trop diversifiés pour permettre de dégager des patterns récurrents. Il aurait probablement fallu examiner des dialogues d'enfants plus jeunes pour en cerner l'émergence.

41 Dans les dialogues étudiés ici le futur simple apparaît de fait de façon préférentielle dans deux types de contextes: la négociation d'activités ou de comportements (23), et l'évocation d'activités ou événements futurs (24). 
(23) Ilona 2;3

Pendant le goûter

ІLо - [liвbаbаь !] <elle montre un livre posé sur la table>.

'lire Babar'

MÈR - nan on va pas lire. on lira après le goûter

(24) Maxime 2;3

MÈR - tu sais où on va ce soir ?

MAX - [pa]

'pas'

MÈR - on va voir Julie

(...)

MÈR - et il y aura Sylvaine aussi

MAX - [kol $\tilde{\varepsilon}]$

'Colin'

MÈR - ouais

MAX - [kol $\tilde{\varepsilon}$ osi]

Colin aussi

MÈr - oui y aura Colin

Dans l'exemple (23), le futur simple est porteur d'une promesse qui fait contrepoint au refus qui, lui, est véhiculé par le futur périphrastique. Ces deux énoncés sont, par ailleurs, en succession temporelle, le refus s'appliquant au moment présent (comme la demande de l'enfant d'ailleurs) alors que la promesse concerne un avenir légèrement distant $d u$ moment de l'énonciation, caractérisé par le changement d'activité (après le goûter). On pourrait penser que l'enchaînement de ces deux énoncés accomplit en même temps un parcours sur la dimension modale/illocutoire et sur la dimension temporelle.

La séquence de l'exemple (24) correspond à un double détachement, à la fois spatial et temporel, de la situation d'énonciation. La mère évoque un déplacement dans l'espace et un moment qui, pour un enfant de deux ans, est nettement dissocié de la situation d'énonciation. Le futur simple, dans un énoncé assertif de type annonce ou prédiction, est ici associé à un verbe existentiel: la mère dépeint à l'enfant l'état de choses qu'il va trouver dans la situation évoquée. Cette anticipation est, d'une certaine façon, préparée par deux énoncés précédents. Dans le premier on trouve un présent à valeur de FUTUR (" tu sais où on va ce soir?»), le second supporte une double interprétation: soit nouvelle occurrence du présent avec valeur de FUTUR sur un verbe de mouvement, soit futur périphrastique (Fleury et Branca-Rosof, 2010). Dans les deux cas ces énoncés, dénotant le déplacement spatial, posent les bases pour l'énonciation d'un état de choses projeté dans le FUTUR et porté par le futur simple. La progression dans le temps semble ainsi ici aussi accomplie par la succession des formes d'expression du FUTUR tout au long de la séquence dialogique ${ }^{15}$.

Ces deux exemples ne font pas exception. Au contraire, il apparaît que parmi les 45 occurrences du futur simple produits par les parents dans le corpus transversal, 38 sont précédées d'une à sept occurrences d'un autre énoncé exprimant le FUTUR. Ces séquences peuvent se dérouler sur un nombre important de tours de parole, comme dans (24) ou se concentrer sur un seul tour de parole, comme dans l'exemple suivant :

(25) Arnaud, 2;3 goûter

MÈr - ça c'est un petit morceau de pomme

ARN - [we ++ kle + mai :: ?] 
'où est ++ Clai(re) +-Ma(r)ie?

MÈR - elle va revenir Claire-Marie, tu finis de manger puis après tu iras la voir. elle est là.

Dans le corpus d'Anaé, on constate également que son frère ainé (âgé de 9 ans) mobilise, comme sa mère, le futur simple en clôture d'une séquence, quand il s'adresse à Anaé :

(26) Anaé $2 ; 7$

Anaé joue avec son frère ainé, Arthur ART - alors tu prends un couteau tu gardes euh le [/] le [/] le [/] le corps [///] la tête d'homme pour la mettre dans les oubliettes et tu gardes le corps de cheval pour faire euh...+

ANA - ah et ça c'est le cheval ?<Anaé montre le centaure>

MÈr - hum

ART - et tu m(e) fabriques une tête de cheval. et après $t(u)$ auras encore un cheval !

Ici Arthur fait référence au résultat d'un processus qu'il est en train de guider. Les énoncés instructionnels, construits avec le présent, décrivent un parcours qui va de l'imminence de l'action à accomplir (prendre un couteau, etc.) au moment, plus lointain, de l'achèvement de la construction.

Presque toujours, l'énoncé au futur simple clôt la séquence, que celle-ci relève de la négociation d'activités ou de l'évocation d'événements futurs. Quand l'énoncé au futur simple n'est pas en clôture absolue, il est suivi d'une coda, qui peut être une justification ( 24) ou de la réitération d'un énoncé précédant (voir (30) plus bas). Dans un cas, les énoncés suivant le futur simple relancent une nouvelle séquence :

\section{(27) Mathilde 2;4}

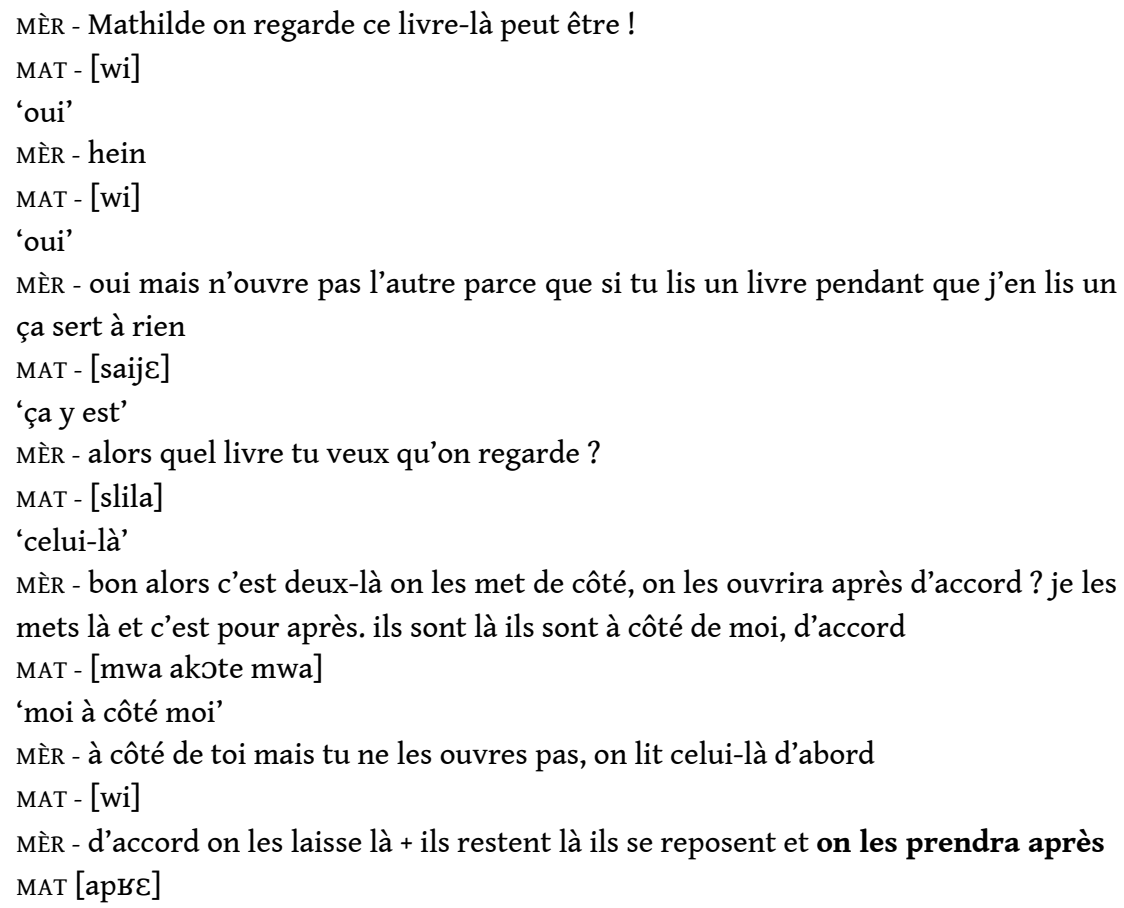

Dans ces enchaînements, la séquentialité temporelle suivante se déploie : les énoncés au présent, dont on a vu le caractère préférentiellement directif, renvoient à un moment quasi contemporain du moment de l'énonciation: c'est le cas de "tu finis de manger " dans l'exemple (25) (voir aussi (11) plus haut). La temporalité des énoncés au futur 
périphrastique est plus indéterminée : elle peut correspondre à un moment ultérieur mais proche du moment de l'énonciation comme dans «elle va revenir » (25) ou à un moment plus clairement distinct, « on va voir Julie » (24). Enfin, l'action mise en mots par le verbe au futur simple est clairement marquée comme dissociée du moment d'énonciation à la fois par le contexte précédent et par la cooccurrence fréquente d'adverbes comme après, tout à l'heure.

Par ailleurs, quand le futur n'est pas associé à un adverbe temporel, il l'est très souvent à des modaux (28) (29) (30).

\section{(28) Théo 2;3}

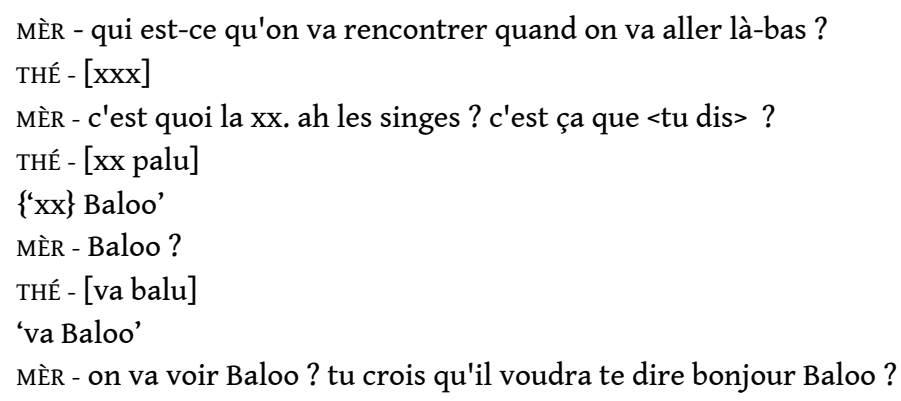

(29) Anaé 3;0

MÈR - Kirikou il est chez mamy Denise.

ANA - mais i(l) faut le reprendre.

MÈR - oui on va le reprendre.

OBS - et tu vas amener tous tes bébés en vacances?

MÈR - XXX. demain [/] demain tu vas chez mamy tu pourras reprendre Kirikou.

(30) Maxime 2;3

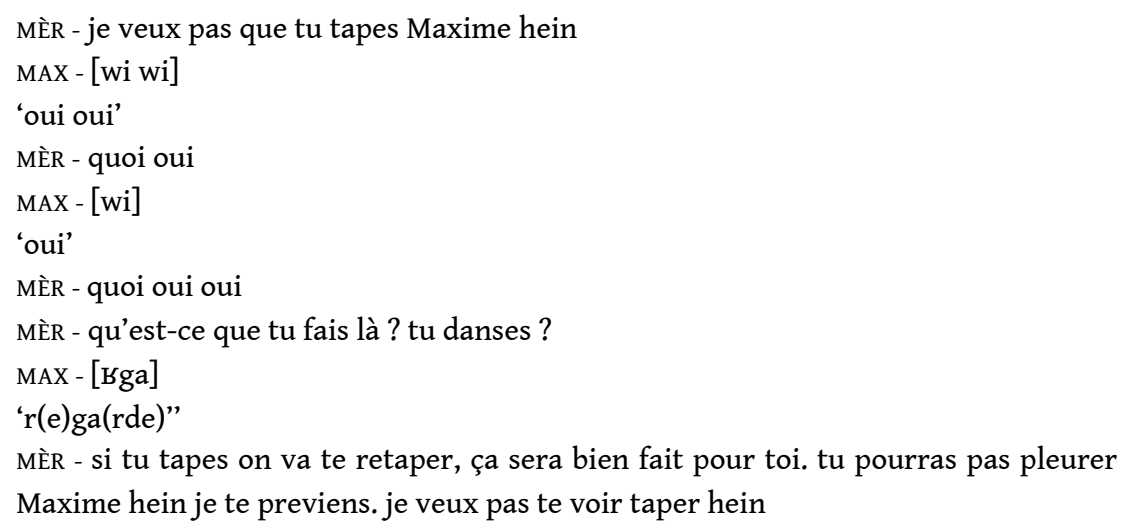

Ces trois exemples suggèrent également que l'usage modal du futur simple est préparé par les énoncés préalables: les mères situent l'univers évoqué dans l'ultériorité du moment de l'énonciation (la visite future à Eurodisney (28), la prochaine visite à des amis (29)) ou dans une temporalité générique (30) et introduisent la modalité au futur simple seulement en un deuxième mouvement. C'est ce dernier type d'énoncé, associant futur simple et modalité, que l'on trouve dans les quelques cas où ce tiroir verbal apparaît de façon isolée dans le dialogue. Il est notable de constater que dans le corpus d'Anaé, quand la mère s'adresse à ses fils plus âgés, elle présente ce type d'usage isolé du futur simple. 
(31) Arthur, frère d'Anaé

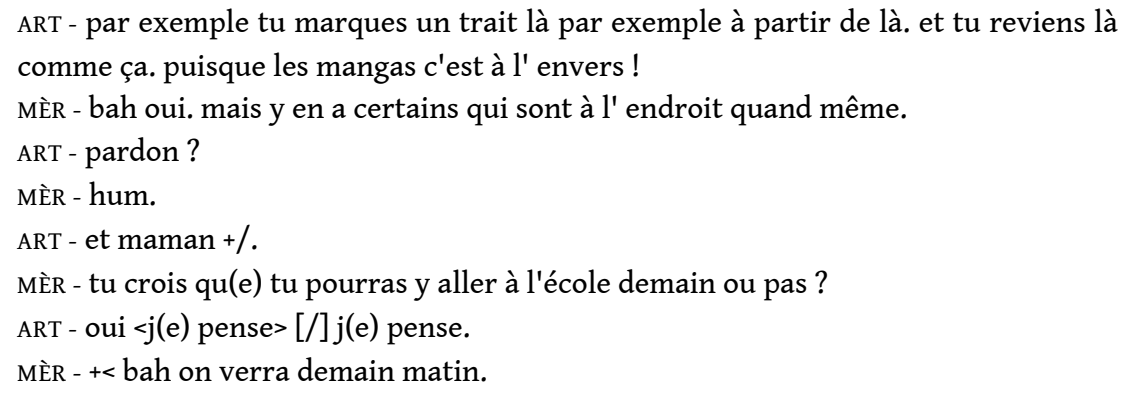

51 L'étude de ce corpus suggère ainsi que les enfants font l'expérience du futur simple dans des contextes dans lesquels les valeurs qui lui sont associées (temporelles ou modales) sont préalablement introduites par les adultes dans l'espace discursif commun. Ce type de séquence pourrait être typique du langage adressé au jeune enfant.

\section{Pour une hypothèse dialogique de l'acquisition du futur}

L'analyse des échanges parents enfants de 24 enfants âgés entre 1;10 et 2;6 a montré que les adultes font référence au FUTUR avec une grande diversité de formes et que les enfants reproduisent (modulo l'absence du futur simple) les grandes tendances des usages adultes. Malgré une certaine hétérogénéité, il apparaît que les enfants font l'expérience, à travers les productions des adultes, de fortes associations entre tiroirs temporels et actes de langage. Compte tenu de l'asymétrie des rôles interactionnels, les enfants reproduisent également ces associations. Enfin, le futur simple, moins fréquent que toutes les autres formes d'expression du FUTUR, est produit par les adultes en fin de séquences dialogiques. On retrouve dans ces séquences les caractéristiques relevées dans les travaux sur la différence entre futur simple et futur périphrastique. Il est notable que grâce à cette façon de faire des adultes, les enfants ne sont pas directement ou abruptement exposés à des formes dont la signification pourrait leur être inaccessible. Sur le plan acquisitionnel, ces séquences présentent toutes les caractéristiques de ce que Bruner (1983), en s'inspirant de Vygotski (2002), appelle l'étayage, processus à travers lequel l'adulte soutient l'activité de l'enfant. L'étayage suppose à la fois la prise en compte du niveau de développement de l'enfant, l'identification de la zone de développement proximal (Vygotski), l'adaptation au niveau de l'enfant en simplifiant la tâche ou en le guidant dans son accomplissement. Dans le cas des séquences impliquant le futur simple, la médiation de l'adulte présente différentes facettes : tout se passe comme si, aussi bien pour les valeurs temporelles que pour les valeurs modales, les adultes (ou les enfants plus grands) préparaient, par étapes successives, la compréhension de l'enfant (et donc son appropriation) des formes. Ces étapes correspondent à une sorte de décomposition ${ }^{16}$ des différents aspects (temporaux, spatiaux, modaux) du détachement de la référence du hic et nunc. Cette "préparation » s'inscrivant dans le dialogue, elle peut même être négociée à travers des questions et des réponses, des ajouts, des reformulations, ce qui assure une intégration de ces éléments dans un espace discursif commun. Quand apparaît le futur simple, l'enfant peut l'intégrer dans du connu ou du maîtrisé, même si cette forme est nouvelle ou moins fréquente. 

dans des contextes de négociation d'activités et comportements, et d'évocation d'actions ou d'événements à venir. Ces séquences présentent dans leur configuration illocutoire et dans leur déroulement les caractéristiques de formats (Bruner, 1987), à savoir des routines interactionnelles dans lesquels l'enfant fait l'expérience de différentes formes de discours et de communication. Par leur répétitivité les formats permettent à l'enfant d'appréhender le sens global de l'interaction en cours et dès lors d'en anticiper le déroulement. Par ailleurs les formats peuvent à leur tour constituer des sous-routines dans le cadre d'échanges plus complexes. C'est le cas de nos séquences qui ne sont pas des activités à part entière, mais qui se développent dans différents types d'activités. Leur configuration récurrente permet probablement aux enfants d'anticiper l'avènement d'un verbe au futur simple.

Bien entendu, l'existence et la fréquence de ce type de phénomène doivent être confirmées dans des données longitudinales exhaustivement étudiées. Il s'agirait alors non seulement d'évaluer leur fréquence dans une population plus large, mais également de vérifier une possible évolution sur le plan diachronique. En amont de la période observée ici, peut-on constater des séquences dialogiques portant sur le FUTUR construites avec des présents et des futurs périphrastiques ? En aval de la période observée ici, quand l'enfant commence à produire lui-même le futur simple, observe-t-on de la part de l'adulte un abandon de ces séquences et un usage direct du futur simple ? Répondre à ces questions permettrait d'étayer l'hypothèse d'une médiation dialogique de l'acquisition du futur.

\section{BIBLIOGRAPHIE}

ABOUDA, L. et SKROVEC, M., 2015, « Du rapport entre formes synthétique et analytique du futur. Etude de la variable modale dans un corpus oral micro-diachronique », Revue de Sémantique et Pragmatique (38), p. 35-57.

ABOUDA, L. et SKROVEC, M., 2017, « Du rapport micro-diachronique futur simple/futur périphrastique en français moderne. Etude des variables temporelles et aspectuelles » Corela. Cognition, représentation, langage. HS-21, http://corela.revues.org/4804.

ALVAREZ CASTRO, C., 2007, «Interprétation du futur de l'indicatif et représentation des événements futurs ", dans L. de Saussure, J. Moeschler et G. Puskas (Eds.), Eudes sémantiques et pragmatiques sur le temps, l'aspect et la modalité, Amsterdam, New York, Rodopi, p. 7-24.

ALVAREZ CASTRO, C., 2010, « Usages temporels et usages modaux du futur en français: dichotomie ou articulation?", La linguistique, 46(2), p. 109-126.

ANTINUCCI, F. et MILLER, R., 1976, « How children talk about what happened ", Journal of Child Language, 3(02), p. 167-189.

AUSTIN, J. L., 1970, Quand dire c'est faire, Seuil, Paris. 
AZZOPARDI, S., 2011, Le futur et le conditionnel ; valeur en langue et effets de sens en discours. Analyse contrastive espagnol/français. Thèse de doctorat Montpellier, Université Paul Valéry-Montpellier III.

BASSANO, D., LAAHA, S., MAILLOCHON, I. et DRESSLER, W. U., 2004, « Early acquisition of verb grammar and lexical development ; Evidence from periphrastic constructions in French and Austrian German », First Language, 24(1), p. 33-70.

BERNICOT, J., 2000, «La pragmatique des énoncés chez l'enfant », dans M. Kail et M. Fayol (Eds.), L'acquisition du langage, Tome II, Paris, PUF, p. 45-82.

BERNICOT, J. et LAVAL, V., 2004, « Speech acts in children; The example of promises ", dans I. A. Noveck et D. Sperber (Eds.), Experimental pragmatics, Basingstoke, UK Palgrave, p. 207-227.

BRANCA-ROSOFF, S., 2009, « Corpus et recherches linguistiques sur le futur simple et le futur périphrastique ». Communication lors du colloque de l'Association for French Language Studies AFLS 2009. Université de Neuchâtel.

BRONCKART, J.-P., 1996, Activité langagière, textes et discours. Pour un interactionnisme socio-discursif, Delachaux et Niestlé., Lausanne.

BRONCKART, J.-P., KAIL, M. et NOIZET, G. (Eds), 1983, Psycholinguistique de l'enfant ; recherches sur l'acquisition du langage, Delachaux et Niestlé, Neuchâtel.

BRONCKART, J.-P. et SINCLAIR, H., 1973, « Time, tense and aspect », Cognition, 2(1), p. 107-130.

BRUNER, J. S., 1983, Le développement de l'enfant. Savoir faire, Savoir dire, P.U.F., Paris.

BRUNER, J. S., 1987, Comment les enfants apprennent à parler, Retz, Paris.

DE WECK, G., 1991, La cohésion dans les textes d'enfants. Etude du développement des processus anaphoriques, Delachaux et Niestlé, Neuchâtel.

DRESSLER, W. U., KILANI-SCHOCH, M. et KLAMPFER, S., 2003, « How does a child detect morphology? Evidence from production », dans W. Bisang, H. H. Hock et W. Winter (Eds.), Trends in linguistics. Studies and monographs, Berlin/ New York, Mouton de Gruyter, p. 391- 425.

FLEURY, S. et BRANCA-ROSOFF, S., 2010, « Une expérience de collaboration entre linguiste et spécialiste de TAL ; L'exploitation du corpus CFPP 2000 en vue d'un travail sur l'alternance Futur simple/Futur périphrastique ", cahiers AFLS, 16(1), p. 63-98.

FRANÇOIS, F., HUDELOT, C. et SABEAU-JOUANNET, E., 1984, Conduites linguistiques chez le jeune enfant, PUF, Paris.

GOSSELIN, L., 1996, Sémantique de la temporalité en français ; un modèle calculatoire et cognitif du temps et de l'aspect, Duculot, Louvain-la-Neuve.

JAKUBOWICZ, C., 1995, « Grammaire universelle et acquisition du langage », Recherches linguistiques de Vincennes (24), p. 7 - 32.

KERBRAT-ORECCHIONI, C., 2001, Les actes de langage dans le discours. Théorie et fonctionnement, Nathan, Paris.

KÜNTAY, A. et SLOBIN, D. I., 2002, « Putting interaction back into child language; Examples from Turkish », Psychology of Language and Communication, 6, p. 5-14.

LEVY, E. T. et NELSON, K., 1994, «Words in discourse; a dialectical approach to the acquisition of meaning and use ", Journal of Child Language, 21, p. 367-389.

LIEVEN, E. et TOMASELLO, M., 2008, « Children's first language acquisition from a usage-based perspective », Handbook of cognitive linguistics and second language acquisition, p. 168 - 196. 
LUST, B. C., 2006, Child language; Acquisition and growth, Cambridge University Press.

MACWHINNEY, B., 2004, « A unified Model of Language Acquisition », dans J. F. Kroll et A. M. B. De Groot (Eds.), Handbook of Bilingualism ; Psycholinguistic Approaches, New York, Oxford University Press, p. 49-67.

MARCOS, H., SALAZAR ORVIG, A., BERNICOT, J., GUIDETTI, M., HUDELOT, C. et PRÉNERON, C., 2004, Apprendre à parler ; influence du mode de garde, L'Harmattan, Paris.

MORGENSTERN, A. et PARISSE, C., 2012, « The Paris Corpus », Journal of French language studies, 22 (01), p. 7-12.

MORGENSTERN, A. et PARISSE, C., 2012, « Premières formes de conditionnel chez l'enfant », Faits de langues, 40(1), p. $219-222$.

MORGENSTERN, A., PARISSE, C. et SEKALI, M., 2010, « A la source du futur ; premières formes verbales dans les productions spontanées de deux enfants français de 18 mois à 3 ans ", Faits de langues, 33, p. 163-176.

NASHAWATI, S., 2010, Le développement des expressions référentielles chez le jeune enfant; noms et pronoms dans des dialogues mère-enfant. Thèse de Doctorat, Paris, Université Sorbonne Nouvelle Paris 3.

NELSON, K., 2007, Young minds in social worlds ; Experience, meaning, and memory, Harvard University Press, Cambridge, MA.

NINIO, A. et SNOW, C., 1996, Pragmatic Development, Westview Press, Boulder.

OCHS, E. et SCHIEFFELIN, B., 1995, « The impact of language socialisation on grammatical development ", dans P. Fletcher et B. MacWhinney (Ed.^Eds.), The Handbook of Child Language, Oxford, Blackwell, p. 73-94.

PINE, J. M., CONTI-RAMSDEN, G., JOSEPH, K. L., LIEVEN, E. V. et SERRATRICE, L., 2008, « Tense over time ; testing the Agreement/Tense Omission Model as an account of the pattern of tensemarking provision in early child English », Journal of Child Language, 35(1), p. 55-75.

PIZZUTO, E. et CASELLI, M. C., 1994, « The acquisition of Italian morphology ; Implications for models of language development ", dans Y. Levy (Eds.), Other Children, Other Languages ; Issues in the theory of Language Acquisition, Hillsdale, N.J., Laurence Erlbaum, p. 137-187.

SABEAU-JOUANNET, E., 1977, « L'expression de l'organisation spatiale et temporelle, son évolution chez des enfants de 2 à 5 ans ", dans F. François, D. François, E. Sabeau-Jouannet et M. Sourdot, Syntaxe de l'enfant avant 5 ans, Paris, Larousse, p. 193-204.

SALAZAR ORVIG, A., 2003, «L'inscription dialogique du jeune enfant ; évolution, diversité et hétérogénéité », TRANEL 38-39, p. 7-24.

SALAZAR ORVIG, A., 2017, « Dialogue et interaction au cœur de la réflexion sur l'acquisition du langage », TRANEL «13ème colloque de logopédie (en l'honneur de Geneviève de Weck). De l'interagir à l'intervenir... : quelles clés pour la logopédie ?, 66 p. 5-26.

SEARLE, J. R., 1972, Les actes de langage, Hermann, Paris.

SEARLE, J. R., 1979, Sens et expression, Minuit, Paris.

SLOBIN, D. I., 1973, « Cognitive prerequisites for the development of grammar », dans C. A. Ferguson et D. I. Slobin (Eds.), Studies of child language development, New York, Holt, Rinehart \& Winston, p. 175-209. 
TOMASELLO, M., 2003, Constructing a language. A usage-based theory of language acquisition, Harvard University Press, Cambridge, Ma. et Londres.

TOMASELLO, M., 2004, Aux origines de la cognition humaine, Retz, Paris.

VENEZIANO, E., 2001, « Displacement and informativeness in child directed talk », First Language, 21(63), p. 323-356.

VENEZIANO, E., 2003, « The emergence of noun and verb categories in the acquisition of French ", Psychology of Language and Communication, 7(1), p. 23-36.

VINEL, E., SALAZAR ORVIG, A., GENEVIÈVE, D. W., NASHAWATI, S. et RAHMATI, S., soumis, « The Impact of Speech Genres on the Use of Referring Expressions », dans A. Salazar Orvig, G. de Weck, R. Hassan et A. Rialland (Eds.), The acquisition of referring expressions; a dialogic approach.

VYGOTSKI, L. S., 2002, Pensée et langage, La Dispute, Paris.

WILSON, S., 2003, « Lexically specific constructions in the acquisition of inflection in English », Journal of Child Language, 30 (1), p. 75-115.

WITTGENSTEIN, L., 1961, Tractatus logico-philosophicus suivi de Investigations philosophiques, Paris, Gallimard.

\section{NOTES}

1. De toute évidence, au moment où l'enfant produit ses premiers mots, et tant que ne se sont pas mis en place les paradigmes morphologiques et les latitudes combinatoires des lexèmes, il est impossible de parler de verbes (ou de noms). Au mieux il s'agit de candidats à verbes, si on considère le statut des lexèmes dans la langue adulte, ou de proto-verbes, si on les considère comme des précurseurs. Néanmoins, pour ne pas alourdir le texte, et comme beaucoup d'autres auteurs, nous utiliserons le terme " verbe".

2. Le corpus a été réuni à l'occasion d'une autre recherche (DIAREF Acquisition des Expressions Référentielles en dialogue ; approche multidimensionnelle, financée par l'ANR, ANR-09- ENFT055). Les données provenaient de divers projets de recherche antérieurs (Salazar Orvig, 2003; Marcos et al., 2004; Nashawati, 2010; Morgenstern et Parisse, 2012).

3. Par convention, on indique l'âge des enfants de la façon suivante : ans;mois.

4. L'analyse présentée ici ne prend en compte que les énoncés des parents lorsqu'ils s'adressent à leur enfant.

5. Dans cet article la valeur sémantico-référentielle FUTUR (comme temps postérieur au moment de l'énonciation) est indiquée en PETITES CAPITALES et les tiroirs verbaux en italiques.

6. Le calcul de la corrélation de Spearman entre la proportion de références au futur dans le dialogue pour l'adulte et l'enfant et la longueur moyenne des énoncés ( $r: 0.106, d l=29, p=0,689)$ a été calculée par enfant sauf pour les suivis longitudinaux où les enfants présentent différentes valeurs LME par séance.

7. Dans les exemples, les productions des enfants sont transcrites en phonétique. La ligne suivante donne l'interprétation entre guillemets simples. Les accolades indiquent une transcription ou une interprétation incertaine. Deux interprétations concurrentes sont séparées par un /.

8. Les formes autres correspondent soit à l'imparfait dans une proposition conditionnelle ou le subjonctif dans une subordonnée de but. Ces cas, marginaux, ne seront pas abordés ici.

9. L'absence de futur simple chez les enfants n'est pas surprenante vu l'âge des enfants dans ce corpus, pour la plupart inférieur à l'âge d'apparition des premiers usages du futur relevés par Sabeau-Jouannet (1977) ou Morgenstern et al. (2010). 
10. Une exploration des usages du futur périphrastique en fonction du type de séquence discursive (discours sur le ici et maintenant, narration fictionnelle, évocation d'expérience personnelle, cf. de Weck, 1991; Bronckart, 1996 ; Vinel et al., soumis) n'a pas donné de résultat intéressant à exposer ici.

11. Dans le domaine des études sur le langage de l'enfant, de nombreux travaux se sont basés sur la classification proposée par Searle. C'est le cas des travaux de Bernicot et son équipe (Bernicot, 2000). En revanche, une toute autre lignée de travaux pointe les limites de ces catégories quand il s'agit de travailler sur les premiers dialogues enfantins (Ninio et Snow, 1996). La place est limitée dans cet article pour entrer dans cette discussion.

12. F indique la présence d'un filler (Veneziano, 2003), syllabe à statut proto-morphologique, non interprétable comme un morphème pré-verbal ou pré-nominal.

13. La question de la personne en fonction sujet est également fondamentale. Dans cet exemple, l'association $2^{\text {ème }}$ personne et présent induit la valeur injonctive. Tout autre sera l'effet de la $1^{\text {ère }}$ ou de la $3^{\text {ème }}$. Cette question qu'on ne peut pas ignorer (cf. Branca-Rosoff, 2009 ; Fleury et BrancaRosoff, 2010) ne peut cependant pas être traitée, par manque d'espace, dans cet article.

14. Pour cette dernière analyse, le corpus transversal a été complété par l'étude de six séances tirées du corpus longitudinal d'Anaé entre 2;2 et 3;5.

15. Je remercie Sonia Branca d'avoir attiré mon attention sur ce type de séquences lors d'un séminaire du projet Le futur dans les langues romanes de CLESTHIA.

16. On a ici un phénomène proche de ce que Kuntay et Slobin (2002) appellent "variation sets » à travers lesquels l'adulte déploie, dans le dialogue, différentes composantes d'un énoncé et en fournit des paraphrases plus simples facilitant la compréhension de l'enfant et le déroulement de l'interaction.

\section{RÉSUMÉS}

Cet article s'intéresse aux usages du futur chez les adultes s'adressant à de jeunes enfants et aux conditions dialogiques qui pourraient favoriser son acquisition. L'analyse des échanges parentsenfants (sur un corpus de 24 enfants âgés entre 1;10 et 2;6 ans) a montré que les parents déploient plusieurs tiroirs verbaux pour référer au FUTUR (du présent au futur simple) et que les enfants reproduisent les grandes tendances des usages adultes, sauf pour le futur simple. Les différentes formes utilisées par les adultes présentent des affinités avec des actes de langage spécifiques, affinités que les enfants reprennent dans des limites déterminées par l'asymétrie des rôles interactionnels. Enfin, le futur simple, moins fréquent que les autres formes d'expression du FUTUR, est produit par les adultes en clôture de séquences dialogiques. Dans ces séquences les adultes introduisent progressivement des éléments de signification (modale ou temporelle) dans l'espace discursif commun, évitant ainsi d'exposer les enfants à des formes dont la signification pourrait leur être inaccessible.

This paper deals with the use of future tense by adults speaking with young children and the dialogic conditions that could favor its acquisition. The analysis of parent-child dialogues, (24 children aged between $1 ; 10$ and 2;6) showed that the parents use different tenses to refer to the FUTURE (from present tense to future tense). The children's distribution of verbal forms follows the adult's uses, excepting for future tense. These various forms are used by adults in preferential 
association with specific speech acts. Again, children present similar patterns, within the limitations determined by the interactional-role asymmetry. Finally, future tense, less frequent than the other forms of reference to FUTURE, appears at the end of specific dialogical sequences. Within these sequences, adults progressively introduce modal and/or temporal meanings in the common ground, thus avoiding exposing children to forms whose meaning could be inaccessible at that point of their development.

INDEX

Mots-clés : temps futur, acquisition, dialogisme

Keywords : future tense, acquisition, dialogism

\section{AUTEUR}

\section{ANNE SALAZAR ORVIG}

Université Sorbonne Nouvelle - Paris 3, CLESTHIA 